\title{
Dificultades cognitivas asociadas a la solución de un ejercicio de mecánica de fluidos en un contexto de aprendizaje virtual
}

\author{
Francisco J. Martínez \\ Escuela de Ingeniería Civil, P. Universidad Católica de Valparaíso, Av. Brasil 2147, Chile. \\ (correo-e: francisco.martinez@pucv.cl)
}

Recibido Jun. 17, 2020; Aceptado Ago. 14, 2020; Versión final Oct. 5, 2020, Publicado Feb. 2021

\begin{abstract}
Resumen
En el presente estudio se examinan las dificultades cognitivas derivadas de una evaluación realizada en un curso avanzado de ingeniería civil en una universidad chilena, en un contexto de aprendizaje virtual. En este contexto y trabajando con un grupo de 70 alumnos, se realiza una tarea de mecánica de fluidos cuya demanda cognitiva es variable. A partir de un análisis esencialmente cualitativo, los resultados muestran que los alumnos logran responder con asertividad cuando la evaluación les plantea desafíos de baja demanda cognitiva. Sin embargo, serias falencias afloran cuando se les apela a construir razonamientos más profundos que involucren el despliegue de habilidades para relacionar conceptos y estructurar una solución de mayor demanda cognitiva. Se concluye que aún en años avanzados de la carrera persisten lagunas formativas que no logran ser resueltas en la transición desde la educación secundaria a la etapa universitaria.
\end{abstract}

Palabras clave: problemas cognitivos; mecánica de fluidos; aprendizaje virtual; ingeniería civil; pensamiento abstracto

\section{Cognitive difficulties in solving a fluid mechanics exercise in a virtual learning environment}

\begin{abstract}
The present study examines cognitive problems observed in higher-level civil engineering courses in a virtual learning context at a Chilean university. A fluid mechanics exercise with variable cognitive demand is assigned to 70 students and their answers are analyzed qualitatively and quantitatively. The results show that students are able to provide correct answers when a task presents challenges of low cognitive demand. However, serious difficulties arise when students are forced to build deeper thinking and reasoning that involves high cognitive intensity and skills capacities in mathematical and physical thinking. It is concluded that, even in advanced engineering courses, cognitive gaps persist that cannot be resolved in the transition from high school to university.
\end{abstract}




\section{INTRODUCCIÓN}

El complejo escenario social y sanitario por el que atraviesa Chile actualmente, ha repercutido en el quehacer de las universidades especialmente desde el segundo semestre del periodo académico 2019/2020 en adelante. Dicho impacto se ha traducido en el impulso que varias casas de estudio le han dado al uso de metodologías y plataformas de aprendizaje virtual para dar continuidad a las actividades académicas, lo cual a la luz de la experiencia internacional trae consigo varios desafíos desde el punto de vista docente, especialmente en cursos de ingeniería (Rahman, 2017). Si bien la definición de aprendizaje virtual se adecúa al contexto en el que se realiza, de acuerdo a Area y Adell (2009) este concepto (también conocido como eLearning) responde a una modalidad de enseñanza-aprendizaje consistente en el diseño, puesta en práctica y evaluación de un curso o plan formativo desarrollado a través de redes computacionales que dan lugar a una formación que se ofrece a un grupo de individuos geográficamente dispersos o separados o que interactúan en tiempos diferidos del docente, empleando para ello recursos informáticos y de telecomunicaciones. En consecuencia, en este escenario el aprendizaje y las interacciones entre los alumnos y el profesor (o tutor) ocurren en un espacio virtual.

En este sentido varios elementos resultan entonces interesantes de relevar en este tipo de entornos. Por un lado, la vivencia relacionada con el desempeño académico de los estudiantes bajo una modalidad donde se reduce significativa, sino totalmente el contacto presencial con los alumnos y, por otro lado, relevar las dificultades cognitivas observadas en el aprendizaje esperado de alumnos de nivel superior en carreras que enfatizan fuertemente el conocimiento basado en la física y las matemáticas. En el presente artículo, entenderemos por dificultad cognitiva a todo aquel problema u obstáculo que altera la capacidad de la memoria, la percepción y la capacidad de resolver problemas.

De acuerdo a Zuñiga-Silva (2018) es importante analizar con detención estas dificultades, sobre todo porque en trabajos de evaluación de competencias se parte de la base que el funcionamiento cognitivo de los alumnos va acorde con la supuesta madurez mental que deberían tener en función de su edad cronológica, aspecto que no necesariamente se cumple en la práctica. Citando otros autores, Zuñiga-Silva (2018) profundiza sobre este punto relevando la necesidad de investigar sobre las funciones y procesos mentales involucrados en el aprendizaje en carreras de alta complejidad, pues aun cuando pueden intervenir factores asociados a los hábitos, creencias, costumbres y otros de índole socio-cultural, este aprendizaje es finalmente resultado de procesos y operaciones mentales internas que dependen de un funcionamiento cognitivo predominantemente individual.

En este contexto se inserta el curso de Mecánica de Fluidos, que forma parte del eje disciplinar de pregrado de la carrera de ingeniería civil, impartido típicamente en el quinto o sexto semestre del plan de estudios en universidades chilenas, aunque esto último depende también de la duración de la carrera. Al cabo del tercer año de estudios, típicamente los alumnos ya han aprobado los cursos obligatorios de ciencias básicas (e.g. cálculo, algebra, física general y mecánica), cuyos contenidos se vuelven indispensables para desarrollar la asignatura. Para este curso la bibliografía de apoyo a la docencia es abundante y si bien, hasta hace algunas décadas varios textos de referencia en el área se encontraban escritos en lengua inglesa y otras, hoy en día varios de ellos ya se han traducido al español (White,2016). A esta lista también se han sumado algunos esfuerzos nacionales ya disponibles comercialmente como por ejemplo Fernández (2013), junto a una lista aún más larga de apuntes de curso fruto de experiencias particulares de docentes universitarios, los que pueden encontrase fácilmente en sitios online de búsqueda (Google).

Un punto común de la bibliografía de mecánica de fluidos se relaciona con la gran cantidad de problemas (con solucionario) que estos libros ofrecen a distintos niveles de complejidad. Este formato se fundamenta en la metodología de aprendizaje orientado a la solución de problemas (based-problems learning). De acuerdo a Kassymova et al. (2020), esta metodología significa en la práctica que los estudiantes son desafiados a aplicar su conocimiento a problemas de la vida real, necesitando replantear, repensar y re-encuadar estos problemas para identificar nuevas soluciones, a diferencia de exámenes o evaluaciones de corta duración, donde a los alumnos típicamente se les conmina a dar una única respuesta posible. De acuerdo a la propia Kassymova, este método de enseñanza continúa estando plenamente vigente en la ingeniería actual y aunque se han detectado problemas relacionados con la mecanización de técnicas, como en cursos de matemáticas (Zuñiga-Silva, 2007), este tipo de aprendizaje es una de las vías importantes que permitirán preparar los futuros profesionales ante la inminente penetración en la era de la inteligencia artificial.

Si bien el formato que ofrecen estos textos facilita el rol docente, los ejercicios que allí se proponen pueden resultar similares de un texto a otro, tanto en sus contenidos, como en nivel de dificultad, incluso sin mayores variaciones entre distintas ediciones. Más que una debilidad, esta característica es vista aquí como una oportunidad para innovar en el diseño de nuevos problemas, que busquen no solo profundizar los conocimientos vistos en clases, sino además abordar dificultades cognitivas y potenciar las competencias asociadas al pensamiento físico y matemático abstracto y la capacidad de relacionar conceptos y estructurar 
razonamientos más profundos. En la bibliografía encontramos abundante información que describe este tipo de dificultades especialmente en cursos de introductorios de formación universitaria, particularmente en el campo de las ciencias básicas (Zuñiga-Silva, 2006 y 2018). Sin embargo, coexiste una realidad opuesta cuando se trata de evaluar estas dificultades de aprendizaje en cursos donde los alumnos se vean conminados a aplicar los conocimientos de ciencias básicas en un contexto más realista y de mayor complejidad que no se limite a la mera aplicación de una fórmula, propio de los cursos de especialidad de la carrera de ingeniería civil.

Así, el presente estudio describe por primera vez dentro de la unidad académica la experiencia docente que ha resultado de la aplicación de un ejercicio de carácter analítico planteado a un grupo de estudiantes de tercer año de Ingeniería Civil que cursan la asignatura de Mecánica de Fluidos en una modalidad de aprendizaje virtual. Atendidas las capacidades esperadas de los alumnos para esta etapa formativa, el objeto del presente estudio es documentar y analizar el desempeño de los estudiantes, entendido este como el nivel de asertividad con que éstos logran abordar el ejercicio, apelando a distintas demandas cognitivas.

\section{METODOLOGÍA}

En el presente artículo se describe un estudio de caso, consistente en un ejercicio analítico planteado a un grupo de aproximadamente 70 estudiantes, de los cuales 59 eran hombres y 11 mujeres y cuyas edades fluctuaban entre 20 y 22 años. El formato de evaluación fue tipo Tarea a ser realizada preferentemente en binomios, sin discriminar aquellos alumnos que preferían trabajar solos. En lo que sigue, entenderemos por Tarea aquella actividad que se lleva a cabo en la clase y que dirige la atención de los estudiantes para desarrollar una idea particular (García y Benítez, 2013). Siguiendo esta definición, la presente tarea ha sido diseñada en el espíritu de promover el desarrollo de competencias genéricas y de competencias específicas en mecánica y física aplicada a la comprensión de la estática de fluidos usando un problema aplicado de ingeniería.

La escogencia de este formato evaluativo se basa en las buenas experiencias reportadas por diversos autores, tales como Báez et al. (2017) y García y Benítez (2013) quienes han plasmado los resultados pedagógicos obtenidos a partir de estos instrumentos de evaluación trabajando en el ámbito de las ciencias matemáticas con alumnos de primeros años de formación universitaria. En paralelo, varios autores han ido un poco más lejos reportando experiencias docentes en cursos de mecánica de fluidos bajo diversos esquemas evaluativos que involucran ejercicios de carácter teórico, de terreno y sobre todo experiencias de laboratorio. Una visión común que se desprende de la mayor parte de estos estudios es que el trabajo colaborativo es un mecanismo valioso para evaluar el aprendizaje basado en competencias, promoviendo la discusión y pensamiento crítico entre pares (Absi et al., 2011; Soares et al., 2015; Huilier, 2019; Pal (2019).

El plazo para desarrollar la tarea fue de un máximo de 7 días corridos. Dicho período se encuentra, por un lado, dentro de los rangos típicos manejados en la unidad académica para evaluaciones de este tipo y, por otra parte, fue considerado un plazo suficiente para que los estudiantes pudieran consultar el material online que les fue facilitado y, sobre todo, favorecer el trabajo colaborativo con otros compañeros del curso usando la plataforma virtual de la universidad. Esta plataforma es un portal a través del cual docente y alumnos pueden interactuar, se puede alojar material docente, evaluaciones previas y habilitar foros de discusión de temas generales o particulares relacionados con el curso. Para esta evaluación el docente habilitó un canal de entrega de la tarea en dicha plataforma y un foro de consultas para los estudiantes, sin restricciones de ningún tipo, con el fin de incentivar su participación durante el plazo de desarrollo del ejercicio facilitando, además, la supervisión docente de carácter virtual.

La posterior recolección y evaluación de datos se realizó luego de la revisión de las hojas de respuestas digitalizadas (escaneadas) por los propios alumnos. Esta evaluación tomo en cuenta aspectos cualitativos y cuantitativos. Los primeros, fueron analizados tomando en cuenta algunos extractos de las respuestas proporcionadas por los alumnos, juzgados por el autor como representativos de las falencias y dificultades típicamente encontradas en dichas respuestas. Desde el punto de vista cuantitativo, este análisis se condujo construyendo un índice de desempeño muy simple que mide, desde la perspectiva del autor, el grado de asertividad en las respuestas entendido en términos de porcentaje de similitud respecto de la pauta de cotejo elaborada por el propio equipo docente.

\section{Posicionamiento teórico}

El análisis de habilidades y/o dificultades cognitivas de las respuestas se inspira significativamente en las directrices entregadas por Zuñiga-Silva $(2007,2018)$, además de los hallazgos reportados por Aznar y Laiton (2017) y particularmente de Rahman (2017) quien ha trabajado con sistemas de aprendizaje on-line en cursos relacionados con la mecánica de fluidos. Siguiendo estos lineamientos, la identificación de dificultades del aprendizaje se encuentra íntimamente ligado con el funcionamiento cognitivo de los estudiantes. De acuerdo 
a Zuñiga-Silva (2018), dicho funcionamiento se puede evaluar tomando como soporte la teoría de las funciones cognitivas de Feuerstein, la que ofrece un catálogo de funciones cognitivas que los seres humanos ponemos en juego al realizar actos mentales de aprendizaje. Siguiendo esta teoría, el procesamiento de información ante cualquier problema que nos enfrentemos se descompone en tres fases: una fase de entrada, una fase de elaboración y una de salida, que aquí describimos brevemente. En la fase de entrada, la comprensión del problema implica poseer una percepción clara del mismo, lo que abarca tanto la comprensión del enunciado, como de la meta que persigue la evaluación. En la fase de elaboración en cambio, la resolución exige la búsqueda de una vía posible de solución, es decir, un camino que conecte el estado inicial con la meta perseguida. Dicha conexión solo tendrá éxito, si el sujeto es capaz de percibir y entender con precisión el problema. Por último, la fase de salida del problema implica que la respuesta del sujeto debe emitirse empleando un lenguaje claro y preciso, de acuerdo a la meta del problema planteado. Tomando en cuenta estas etapas, se realizó el diseño del ejercicio cuyas características se describen a continuación.

\section{Planteamiento y estrategia de solución del ejercicio}

El problema planteado fue diseñado por el autor del presente artículo. A través de él, no se pretendía presionar desmesuradamente a los estudiantes con contenidos fuera de la asignatura sino, por el contrario, buscaba situar a los estudiantes fuera del espectro de problemas a los que típicamente acceden en los textos clásicos de la materia como fue señalado anteriormente. Dicho planteamiento, además, busca evitar que los alumnos puedan repetir o mecanizar soluciones de problemas plagiando respuestas obtenidas desde dichos textos de referencia u otros medios difundidos en la web.

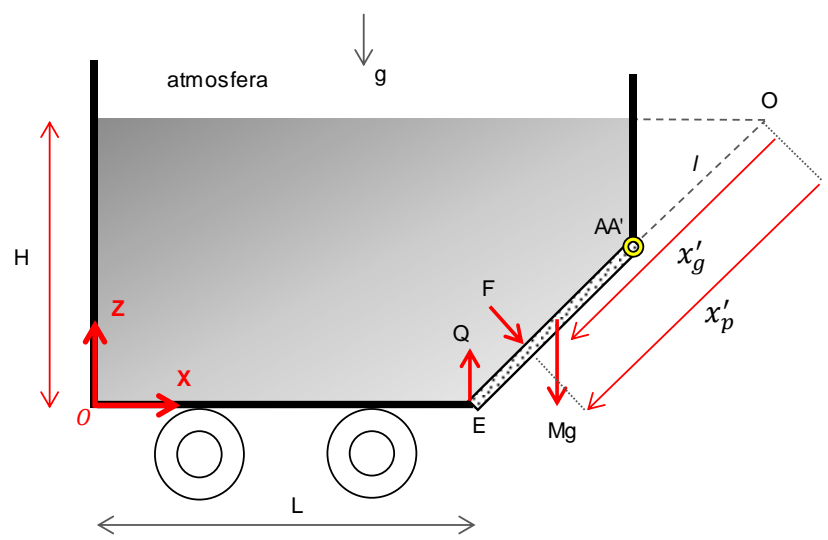

a)

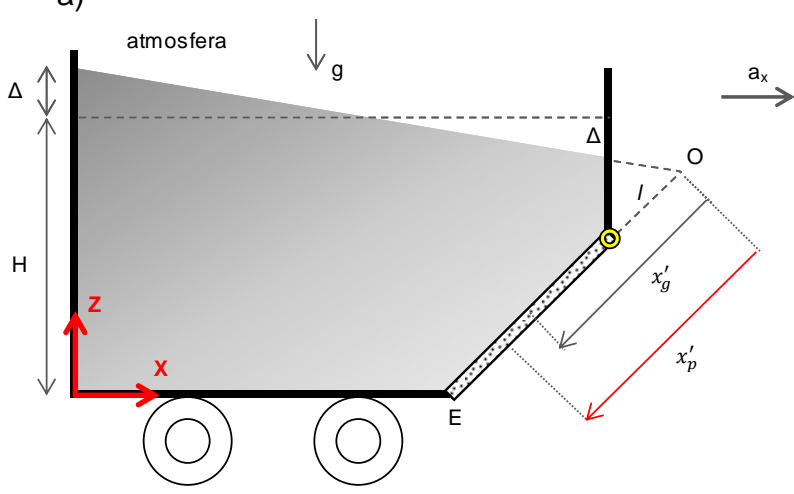

c)

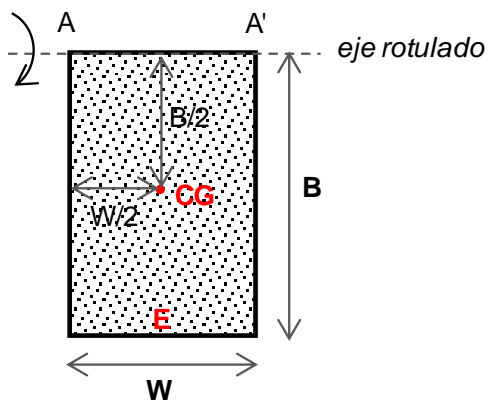

b)

Fig. 1. Esquemas usados para el desarrollo del problema. a) Carro en la situación inicial sin aceleración; b) Geometría de la placa sumergida c) Deformación del líquido una vez el carro acelera bajo una aceleración

La Figura 1 muestra esquemas usados para el desarrollo del problema. a) Carro en la situación inicial sin aceleración, donde se identifica la fuerza hidrostática $F$ y la fuerza $Q$ aplicadas sobre la placa sumergida. En el mismo esquema se define el origen del sistema de coordenada longitudinal, donde $x_{g}^{\prime}$ y $x_{p}^{\prime}$ denotan la posición del centro de gravedad y de presiones., respectivamente. En el mismo esquema se muestra el sistema de coordenadas respecto del cual se calcula la distribución de presiones. b) Esquema mostrando la geometría de la placa sumergida, donde $W$ es el ancho y $L$ la longitud y c) esquema mostrando la deformación del líquido una vez el carro acelera bajo una aceleración $a_{x}>0$. Aquí $\Delta$ denota la sobreelevación del fluido en las paredes verticales.

En este contexto, el problema que se plantea a continuación profundiza el concepto de fuerzas hidrostáticas sobre cuerpos sumergidos y fuerza al alumno a buscar sus propias metodologías que permitan cuantificar 
esta dinámica en situaciones menos familiares, invitándolos a cuestionarse hasta qué punto es posible extender las leyes de la hidrostática en sistemas no inerciales. Las Figuras 1a y 1c esquematizan el problema, mostrando las fuerzas que participan del fenómeno y las variables geométricas tanto para la condición estática, como uniformemente acelerada. El ejercicio trata entonces con el cálculo de fuerzas hidrostáticas sobre cuerpos sumergidos y considera un estanque tridimensional de ancho B y cuyas otras dimensiones se muestran en la Figura 1a. Este estanque contiene agua en su interior ocupando un cierto volumen inicial. En una esquina del fondo del estanque se ubica una compuerta sólida de características físicas y geométricas conocidas como muestra la Figura 1b. Esta compuerta se sella en su extremo inferior en el punto E, pero puede girar alrededor del eje rotulado AA como muestran las Figuras 1a y $1 \mathrm{~b}$. Sobre este sello se engendra una fuerza vertical ascendente (a determinar) que impide la apertura de la compuerta a todo evento. Repentinamente el estanque acelera horizontalmente hacia la derecha bajo una aceleración constante y positiva.

Varias preguntas surgen naturalmente y que queremos responder: ¿qué fuerza es necesaria aplicar sobre el sello de la compuerta para que no filtre el estanque, tanto para el escenario de reposo absoluto mostrado en la Figura 1a, como el caso uniformemente acelerado observado en la Figura 1c? ¿cuál de ambas situaciones (reposo absoluto vs. escenario acelerado), resulta más favorable para abrir la compuerta? En este esquema de trabajo las respuestas de los alumnos fueron cotejadas con la pauta de solución que se detalla en la sección de a continuación, que para mayor comodidad se ha dividido en dos partes.

\section{Carro en situación de reposo absoluto (Parte I)}

En la situación de reposo absoluto, los alumnos deben identificar las fuerzas actuando sobre la placa sumergida, siguiendo el esquema mostrado en la Figura 1a. En dicho esquema distinguimos la fuerza resultante del campo de presiones (fuerza hidrostática), la fuerza vertical sobre el sello de la compuerta y el peso propio de ésta. Aunque no están indicadas en este esquema, los alumnos también deben considerar las reacciones que surgen en la rótula las que, sin embargo, no intervienen cuando se realiza la conservación de torque alrededor de dicho punto. Este es el primer paso esencial que los alumnos deben realizar en su proceso cognitivo, es decir, comprender el enunciado e identificar variables esenciales del problema. De acuerdo a Fox (2003), la determinación de la presión hidrostática para un fluido en reposo absoluto se calcula como:

$$
\frac{d P}{d z}=-\rho g \text {, donde } \rho \text { es la densidad del fluido y } g \text { es la gravedad }
$$

Para conocer la presión, la ecuación (1) se debe integrar en la profundidad del estanque, conduciéndonos a la relación:

$$
P=-\rho g z+C \text {, donde } \mathrm{C} \text { es una constante }
$$

Los estudiantes deben identificar que la distribución (2) no es completa, debiendo precisar la constante que allí interviene, la que se evalúa según las condiciones de borde. De acuerdo al sistema de referencia de la Figura 1, la presión en la superficie libre del fluido corresponde a la presión atmosférica la que puede considerarse nula si trabajamos en un esquema de presiones relativas. Estos conceptos fueron enseñados a los alumnos con anterioridad en las clases virtuales, junto al material docente alojado en la plataforma virtual y las ayudantías del curso realizadas por el equipo docente. Con esto se tiene:

$$
C=\rho g h \text { y } P=\rho g(h-z) \text {, donde } P \text { es la presión relativa }
$$

En este marco conceptual, los estudiantes pueden determinar la fuerza hidrostática sobre la placa que corresponde a la integración del campo de presiones. Este punto es importante, pues algunos estudiantes se ven tentados a reducir esta fuerza al simple producto de la presión por la superficie de la placa, desconociendo con ello el carácter variable de la presión punto a punto de la superficie. Con esto se tiene:

$$
\vec{F}=\iint_{S} P \hat{n} d S=\iint_{S} \rho g(h-z) \hat{n} d x^{\prime} d y^{\prime}=\rho g h S \hat{n} \text {, donde } \hat{n} \text { es un vector normal unitario }
$$

A partir de la ecuación (4) se obtiene el valor absoluto de la fuerza y las variables que en ella intervienen:

$$
F=\gamma h S \text {, donde } S=B W \text { y } h=H-\frac{B}{2} \sin \alpha \text {, donde } S \text { es el área de la placa }
$$

Siendo $h$ la profundidad del centro de gravedad de la placa medida desde la superficie libre. Un problema común de los estudiantes en esta etapa es reconocer que la fuerza dada por la fórmula (5), por una parte, actúa perpendicular a la placa como consecuencia de la naturaleza del campo de presiones y, por otro lado, actúa en un punto denominado centro de presiones cuya coordenada la denotaremos por: 


$$
\overrightarrow{r_{p}^{\prime}}=\left(x_{p}^{\prime}, y_{p}^{\prime}\right)
$$

Como parte de los contenidos vistos en clases, se sabe, salvo que la placa sea perfectamente horizontal, que el centro de presiones se ubica más abajo que el centro de gravedad, como se desprende de la ecuación (7):

$$
x_{p}^{\prime}=x_{g}^{\prime}+\frac{I_{G}}{x_{g}^{\prime} S} \text {, donde } x_{g}^{\prime}=l+\frac{B}{2} \text { es la coordenada del centro de gravedad e } I_{G}=\frac{W B^{3}}{12}
$$

El centro de gravedad se mide aquí tomando como origen la intersección de la proyección de la superficie libre con la proyección del eje longitudinal de la placa, como muestra la Figura 1a. Por último, también se enseñó a los alumnos que en cuerpos simétricos el producto del momento de inercia se anula y el sistema de coordenadas siempre se puede escoger de forma tal que:

$$
y_{p}^{\prime}=y_{g}^{\prime}=0
$$

Por lo tanto, los alumnos en esta primera etapa no deberían tener dificultades mayores en reconocer que la ecuación (8) permite entonces reducir dos grados de libertad el problema. Conocidos entonces las fuerzas y puntos de aplicación, la determinación de la fuerza sobre el sello se apela a dos condiciones simultáneas que aseguran el equilibrio de la placa: por un lado, la ecuación de equilibrio de fuerzas y, por otra parte, la ecuación de conservación de torque. Esta última se reduce:

$$
(F) x_{p}^{\prime}-(Q \cos \alpha) B+(W \cos \alpha) \frac{B}{2}=0 \Rightarrow Q=\frac{W}{2}+\frac{\gamma h S x_{p}^{\prime}}{B \cos \alpha}
$$

La ecuación (9) se convierte en un resultado concluyente de la primera parte de la tarea y llegar a ella debería ser el resultado natural de la aplicación de conceptos introductorios del curso, sumados a contenidos vistos en cursos introductorios de Fisica de cuerpos en equilibrio mecánico. Si bien ninguno realizó este alcance, como veremos más adelante, los alumnos también podrían notar que la relación (9) solo es una expresión que la fuerza sobre el sello balancea el peso propio de la placa y del fluido proyectado sobre ella. En la sección que mostramos a continuación es donde los estudiantes son conminados a realizar un razonamiento más profundo y donde deben mostrar habilidades para deducir su propia formulación para encontrar la fuerza sobre el sello en un sistema uniformemente acelerado.

\section{Carro animado de un movimiento uniformemente acelerado (Parte II)}

Cuando el carro comienza a acelerar, la superficie libre del fluido contenido al interior del estanque se deforma siguiendo la geometría mostrada en la Figura 1c. En esta nueva condición, los alumnos deben notar que participan las mismas fuerzas que las indicadas en la Figura 1a pero sus magnitudes cambian, excepto la del peso propio de la compuerta. Para encontrar estas nuevas magnitudes debemos resolver el campo de presiones en esta nueva situación acelerada planteando la ecuación diferencial siguiente (White, 2016):

$$
\vec{\nabla} P=\rho \vec{f}_{m}
$$

En clases fue visto que el campo de aceleraciones externo se superpone al campo gravitacional, dando como resultado el siguiente vector resultante de aceleraciones:

$$
\vec{f}_{m}=-\rho a_{x} \hat{\imath}-\rho g \hat{k}
$$

La integración de las fórmulas (10) y (11) nos conduce a:

$$
P(x, z)=-\rho a_{x} x-\rho g z+C, \text { donde } C=\gamma(H+\Delta) \text { y } \Delta=\frac{1}{2}\left(\frac{a_{x}}{g}\right)(L+B \cos \alpha)
$$

En el enunciado se indica a los alumnos que asuman que el estanque es lo suficientemente grande para no tener problemas de rebase del fluido. Con la expresión (12) se tiene completamente determinado el campo de presiones en cualquier punto del fluido y con ello podemos determinar la fuerza hidrostática sobre la placa. Esta etapa entraña una demanda cognitiva importante, pues los alumnos deben reconocer que para obtener dicha fuerza (una de las metas del ejercicio) se debe apelar nuevamente a la ecuación (4). Entremedio, los alumnos deben notar que el elemento diferencial de superficie está orientado en un plano parametrizado por coordenadas distintas de las que caracterizan el campo de presiones, lo que obliga a realizar algunas operaciones trigonométricas para relacionar ambos sistemas de referencia. Este proceso conduce a la fuerza hidrostática: 


$$
F=-\rho a_{x} S\left(L+\frac{B}{2} \cos \alpha\right)-\frac{1}{2} \rho g B S \sin \alpha+C \cdot S
$$

El siguiente paso es determinar la posición del centro de presiones. Aquí es necesario observar que la fórmula (7) se revela insuficiente, pues ha sido determinada asumiendo que la distribución de presiones viene dada por la ecuación (3) y no la ecuación (12). Por lo tanto, los alumnos deben apelar a la metodología que nos condujo a ecuación (7) para obtener una nueva fórmula para este caso. Este paso es tal vez el más difícil de mayor demanda cognitiva en todo el ejercicio y que requiere haber madurado los contenidos del curso en su etapa introductoria. Como veremos más adelante en la presentación de resultados, muy pocos alumnos repararon en esta observación y asumieron a rajatabla la fórmula (7) sin cuestionarse las hipótesis que la sustentan. Siguiendo entonces este razonamiento, el punto cognitivo clave es reconocer y comprender que la determinación del centro de presiones surge de un balance de torque sobre la placa, esto es:

$$
\vec{F} \times \overrightarrow{r_{p}^{\prime}}=\iint_{S} P(x, z) d S\left(\hat{n} \times \overrightarrow{r^{\prime}}\right) \Rightarrow\left\|\vec{F} \times \overrightarrow{r_{p}^{\prime}}\right\|=F x_{p}^{\prime}=\iint_{S} P(x, z) x^{\prime} d S
$$

Del desarrollo algebraico de la ecuación (14) es posible despejar la componente longitudinal del centro de presiones:

$$
x_{p}^{\prime}=\frac{-\rho a_{x}\left\{(L+(B+l) \cos \alpha) x_{g}^{\prime} S-\cos \alpha\left(I_{G}+S x_{g}^{\prime 2}\right)\right\}-\rho g\left\{B \sin \alpha S x_{g}^{\prime}-\sin \alpha\left(I_{G}+S x_{g}^{\prime 2}\right)+l \sin \alpha S x_{g}^{\prime}\right\}+C \cdot S x_{g}^{\prime}}{\rho a_{x} S\left(L+\frac{B}{2} \cos \alpha\right)-\frac{1}{2} \gamma S B \sin \alpha+C \cdot S}
$$

Sin duda que la formula (15) luce engorrosa, pero su complejidad demanda elementos cognitivos que no van más allá de los adquiridos en un curso introductorio de álgebra o cálculo. Otro elemento importante que los estudiantes siempre deben tener en cuenta es que toda expresión engorrosa invita a realizar una verificación de su certitud, considerando que la probabilidad de cometer errores algebraicos en su deducción evidentemente aumenta. En consecuencia, dicha certitud se evalúa fácilmente para la situación no acelerada, en cuyo caso deberíamos recuperar la ecuación (5). Efectivamente, realizando varias manipulaciones algebraicas en esta condición se tiene:

$$
F x_{p}^{\prime}=-\gamma x_{g}^{\prime} S B \sin \alpha+\gamma \sin \alpha\left(I_{G}+S x_{g}^{\prime 2}\right)+\gamma H S x_{g}^{\prime} \Rightarrow F=\gamma h S
$$

La ecuación (16) corresponde a la fuerza que habíamos encontrado en la expresión (5), como habíamos planteado. Finalmente, la Tabla 1 resume la información proporcionada a los alumnos durante el ejercicio y los resultados del mismo.. De estos resultados se desprende que, en una situación de movimiento acelerado,

\begin{tabular}{|c|c|c|c|}
\hline \multirow{2}{*}{ Variable o Parámetro } & \multicolumn{2}{|c|}{ Magnitud } & \multirow{2}{*}{ Comentario } \\
\hline & Parte I & Parte II & \\
\hline$H(m)$ & 2.0 & 2.0 & \multirow{8}{*}{$\begin{array}{l}\text { Información entregada a los } \\
\text { alumnos }\end{array}$} \\
\hline$L(m)$ & 1.5 & 1.5 & \\
\hline$B(m)$ & 1.0 & 1.0 & \\
\hline$W(m)$ & 0.6 & 0.6 & \\
\hline$\rho\left(\mathrm{kg} / \mathrm{m}^{3}\right)$ & 1000 & 1000 & \\
\hline$\rho_{s}\left(\mathrm{~kg} / \mathrm{m}^{3}\right)$ & 7000 & 7000 & \\
\hline$\alpha\left({ }^{\circ}\right)$ & 45.0 & 45.0 & \\
\hline$a_{x}\left(\mathrm{~m} / \mathrm{s}^{2}\right)$ & 0.0 & 5.0 & \\
\hline$\Delta(m)$ & 0.00 & 0.56 & \multirow{5}{*}{$\begin{array}{l}\text { Resultados a determinar en el } \\
\text { ejercicio }\end{array}$} \\
\hline$F(N)$ & $\sim 9681$ & $\sim 7431$ & \\
\hline$Q(N)$ & $\begin{array}{l}7438 \\
\end{array}$ & $\begin{array}{c}6097 \\
\end{array}$ & \\
\hline$x_{g}^{\prime}(m)$ & 2.33 & 1.18 & \\
\hline$x_{p}^{\prime}(m)$ & 2.36 & 1.25 & \\
\hline
\end{tabular}
se reduce la fuerza requerida para mantener bloqueada la compuerta y además se reduce, la fuerza hidrostática aplicada sobre la compuerta, respondiendo las interrogantes propuestas a los alumnos.

Tabla 1. Variables del problema, con sus dimensiones y magnitudes características 


\section{RESULTADOS}

Después de analizar los datos de las diferentes respuestas de los estudiantes, se clasificaron los trabajos de acuerdo a la pauta de desempeño, tomando en cuenta semejanzas en los procedimientos utilizados. Los párrafos extraídos a continuación muestran una forma de trabajo representativa del grupo completo.

\section{Rendimiento cuantitativo de la evaluación}

En la Tabla 2 se muestran los criterios adoptados para evaluar el rendimiento de los estudiantes en la Tarea. Siguiendo la terminología de esta pauta, se considera un desempeño "pobre" cuando la similitud entre la estrategia de respuesta respecto de la pauta de cotejo resulta inferior al 25\%; un desempeño "aceptable" cuando dicho nivel se encuentra entre un $25 \%$ y un 50\%; desempeño "destacado" entre $50 \%$ y $75 \%$ y "sobresaliente", cuando se tiene sobre un $75 \%$ de similitud. Este indicador es meramente subjetivo, pero le permitió al autor clasificar y diagnosticar el desempeño cognitivo de los estudiantes de acuerdo a la meta trazada para el ejercicio.

Siguiendo este criterio, en términos generales la primera parte de la tarea los estudiantes logran abordarla y responderla con destreza, demostrando que los conocimientos básicos de hidrostática han sido bien absorbidos en el curso. Esto se refleja en un $70 \%$ de respuestas aceptables y alrededor de un $14 \%$ de respuestas sobresalientes que muestran un alto nivel de concordancia con la pauta de cotejo. Estos buenos resultados sugieren que los estudiantes del curso logran comprender, desarrollar y dar una salida aceptable al problema cuando la demanda cognitiva propuesta es de baja intensidad o cuando ésta los sitúa en lugares comunes del curso, entendiendo por lugares comunes aquellas problemáticas o desafíos que requieren una aplicación directa de las formulaciones y conceptos vistos en el curso.

En la segunda parte de la evaluación es donde se evidencian los problemas más serios. En esta etapa los estudiantes fueron forzados a una mayor demanda cognitiva, implicando tras ello la determinación varias variables y fuerzas participantes del fenómeno como requisitos para encontrar la fuerza buscada sobre el sello. Para ser más precisos, ya la determinación del campo de presiones hidrostáticas en un escenario acelerado junto con el cálculo del centro de presiones, planteó problemas de cálculo a los alumnos, demostrando con ello que no se logra reconocer la diferencia desde el punto de vista físico, entre una situación de reposo absoluto, respecto de un movimiento acelerado. ¿Porque era importante incluir esta parte como diseño del ejercicio? Porque se espera a través de él que los alumnos logren razonar que la existencia de un campo acelerado que se superpone al campo gravitacional, es capaz de modificar el campo de presiones del fluido y, en consecuencia, es esperable que se modifique la magnitud (aunque no la dirección) de la fuerza hidrostática sobre la placa.

Este razonamiento entraña un proceso cognitivo desafiante y que, al mismo tiempo, debería despertar el interés de los alumnos en tanto profundiza las perspectivas y demandas cognitivas típicamente encontradas en problemas de hidrostática. Ningún alumno reparó en este tipo de razonamiento o al menos, fueron capaces de construir algún comentario que entregara luces de una comprensión de esta nueva situación. Como se observa en la Tabla 2, solo en el $2.9 \%$ de los casos se observaron estrategias aceptables de respuesta que dieron luces al evaluador de algún grado de comprensión del fenómeno. Por cierto, ningún estudiante logró calcular correctamente las anteriores variables y, en consecuencia, tampoco la fuerza buscada.

Por otro lado, aun cuando la complejidad algebraica de las fórmulas (13) y (15) invita a realizar una verificación de su certitud, es preocupante que ningún alumno haya reparado en este chequeo. En base a la experiencia del docente, este aspecto podría ser un indicador de un exceso de confianza en la actitud de los estudiantes al momento de responder, sumado a carencias en el desarrollo de la capacidad de auto-crítica. En esta línea, resulta también llamativo que aun cuando se habilitó un foro de discusión en la plataforma virtual de la universidad, menos de un $10 \%$ de los integrantes del grupo hizo un uso efectivo de ella. Más aún, solo aproximadamente un $5 \%$ del grupo de estudio optó por realizar consultas vía correo electrónico al docente a cargo y otros estudiantes optaron por formular preguntas directamente a los ayudantes del curso, aunque este último aspecto no pudo metrizarse con certeza debido a que estas consultas ocurren en un ambiente no controlado por el docente.

Del resto del grupo se observan imprecisiones, ambigüedades y, sobre todo informalidad en la elaboración de sus respuestas, dejando así la mayor parte del trabajo interpretativo al revisor. Este tipo de conductas que nacen como resultado de la transferencia de conocimientos a un escenario cognitivo menos convencional, ya ha sido reportado por Chandler et al. (2013) trabajando en extensos estudios con miles de estudiantes de EE. UU. De acuerdo a Zuñiga-Silva $(2007,2018)$, estos vicios podrían estar arrastrándose desde la etapa escolar y podrían ser explicados, en principio, debido a conductas egocentristas de los estudiantes que pueden remediarse con una adecuada formación en los dos primeros años de formación universitaria. 
Tabla 2. Desempeño en la evaluación atendiendo al porcentaje de similitud de las hojas de respuesta con la pauta de cotejo.

\begin{tabular}{|l|l|l|l|}
\hline Similitud respecto de la pauta de cotejo & Resultado & Parte I & Parte II \\
\hline$<25 \%$ & Pobre desempeño & $7.1 \%$ & $64.3 \%$ \\
\hline $25 \%$ a $50 \%$ & Aceptable & $71.4 \%$ & $25.7 \%$ \\
\hline $50 \%$ a $75 \%$ & Destacado & $14.3 \%$ & $7.1 \%$ \\
\hline$>75 \%$ & Sobresaliente & $7.1 \%$ & $2.9 \%$ \\
\hline & Total & $100.0 \%$ & $100.0 \%$ \\
\hline
\end{tabular}

Si bien todos los conceptos planteados en la pauta de cotejo fueron vistos en el curso, no deja de ser llamativo que estas demandas cognitivas tampoco lograron ser destrabadas cuando el profesor entregó indicaciones para su desarrollo. A lo anterior, en gran parte de las hojas de respuesta se evidencian dificultades ligadas al desarrollo de operaciones básicas de cálculo diferencial e integral y de razonamiento físico que pueden ser soslayados, usando conceptos de Física General y Mecánica Racional. De la misma Tabla 2, solo alrededor de un $10 \%$ del grupo (grupo "destacado" y "sobresaliente") logra resolver con asertividad la Parte II del ejercicio, dejando en evidencia una fuerte merma en el rendimiento académico ante un aumento moderado o alto en la exigencia cognitiva.

\section{Análisis cualitativo de los reportes}

Para reforzar las ideas anteriores y realizar un análisis desde el punto de vista cualitativo del desempeño de los alumnos, se presentan a continuación cinco extractos de respuestas entregadas por los estudiantes, considerados por el docente como muestras representativas del comportamiento de todo el grupo de estudio y que desnudan los errores conceptuales más comunes encontrados en el desarrollo del ejercicio. A continuación, describimos algunos de ellos.

\section{Extracto 1}

Un alumno incluye un esquema donde muestra la inclinación de la isobara 0 (superficie libre), identifica la profundidad a la cual se ubica el centro de la placa y otra profundidad que aparentemente corresponde al punto medio del estanque. El alumno deja esta interpretación al revisor. El alumno incluye además las siguientes líneas de cálculo, bajo el esquema:

$$
\text { “ } \nabla P=\rho \vec{f}_{m}=\rho(-5 \hat{\imath}-9.8 \hat{\imath}) \Rightarrow \frac{d P}{d x} \hat{\imath}+\frac{d P}{d y} \hat{\jmath}=\rho(-5 \hat{\imath}-9.8 \hat{\imath}) \Rightarrow \frac{d P}{d x}=-5 \rho \quad \text { y } \quad \frac{d P}{d y}=-9.8 \rho "
$$

Algebraicamente, las ecuaciones anteriores están correctamente planteadas y permitirían determinar el campo de presiones en una situación de movimiento acelerado, pero el alumno no entrega mayor argumentación que permite entender cómo es posible pasar de una línea de cálculo a otra. De acuerdo a Zuñiga-Silva (2018), aquí el alumno sí logró una percepción y elaboración relativamente clara del problema, pero encuentra problemas en la fase de salida, esto es, en plantear una respuesta clara y precisa. Esto se ve acentuado, aunque este punto en realidad es un error no tan grave, de confundir el concepto de derivada total con el de derivada parcial más propio de una función multivaluada. Finalmente, tampoco se observa una conclusión del cálculo quedando el análisis incompleto y reafirmando la observación anterior. La figura que usa el estudiante para apoyar su esquema de cálculo tampoco resulta de gran apoyo para el el desarrollo y de hecho esta no interviene, sino en la parte final del ejercicio cuando corresponde hacer el diagrama de cuerpo libre sobre la compuerta sumergida.

\section{Extracto 2}

Un estudiante ya ha determinado el campo escalar de presiones y se apronta a calcular la fuerza necesaria $(Q)$ para que no se abra la compuerta. En su desarrollo, efectivamente, se indica el punto de cálculo de la presión mediante un punto B en un diagrama, sin embargo, no desarrolla el cálculo de la fuerza $F$ que correspondería a la fuerza sobre la placa. Luego de eso el estudiante plantea una ecuación de conservación de torque señalada escuetamente como:

$$
\begin{aligned}
& \text { "ahora evaluando } P \text { en el punto } B\left(x_{p} \cos \alpha, x_{p} \operatorname{sen} \alpha\right) \text {, } \\
& P(B)=\rho_{a x} x_{p} \cos \alpha-\rho_{g} x_{p} \operatorname{sen} \alpha+\rho g h-0.562 \rho_{g} \\
& \int P(B) d S=F \\
& \text { Luego se hace } \sum M_{o} \\
& -F_{e} b \cos \alpha+F x_{p}+P . p l a c a-\frac{b}{2}=0
\end{aligned}
$$


Por un lado, este resultado es al menos curioso en tanto el alumno su logra identificar los pasos que le permitirían eventualmente resolver el problema, pero basado en un esquema de alta informalidad. Es decir, nos topamos nuevamente con problemas de precisión y exactitud en la respuesta, que forman parte de la etapa de salida. Por otra parte, estas falencias sitúan al evaluador en una doble disyuntiva, es decir, debe ser capaz de interpretar benévolamente el hilo conductor propuesto por el alumno y al mismo tiempo presupone que las cantidades físicas no desarrolladas han sido correctamente calculadas por algún otro medio no explicitado en la hoja de respuesta.

\section{Extracto 3}

Un estudiante propone un diagrama de cuerpo libre (DCL) actuando sobre la placa sumergida. Si obviamos las reacciones que actúan sobre el eje rotulado del cuerpo el esquema propuesto por el estudiante reconoce efectivamente las fuerzas actuando sobre el cuerpo, a saber, las fuerzas F, Q y el peso propio del cuerpo. Sin embargo, el estudiante no realiza un esfuerzo mayor en identificar las otras dimensiones características que participan del fenómeno, dejando el diagrama incompleto. Aquí, no solo es un problema de percepción y organización de la información, sino además se pueden incorporar variables motivacionales que podrían estar conduciendo a un desprecio por la actividad evaluativa. En otro esquema del mismo alumno, las fuerzas y variables resultan ininteligibles habida cuenta que se dio el tiempo suficiente para prevenir estos problemas de edición o al menos, para haberlos advertidos al docente a través de los medios virtuales. En ambos casos, los diagramas de cuerpo libre prestan escaso apoyo a la solución del problema invitando a preguntarnos: ¿cuál es el sentido de colocar esos diagramas ahí? Este tipo de esquemas, que se aprenden usualmente desde la educación secundaria y se refuerzan en los cursos introductorios de física, parecen ser realizados sin mayor meditación y dentro de una lógica que parece despreciar su utilidad. Si no se logran reconocer bien las fuerzas participantes de un fenómeno y sus escalas características, podemos preguntarnos legítimamente ¿podrán plantearse correctamente las ecuaciones que conducen a la solución del problema?

\section{Extracto 4}

Un alumno plantea correctamente a través de un esquema una ecuación para la determinación de la fuerza sobre la placa. Sin embargo, extrañamente el estudiante proporciona un argumento falaz para reducir la complejidad algebraica de la integral allí involucrada, del tipo:

$$
\begin{aligned}
& \text { " } F_{p}=\iint_{A} P(x, z) d x^{\prime} d y^{\prime}, y \text { varía entre } 0 \text { y } 6 \mathrm{~cm} \text { un rango bastante mínimo por lo que para efectos del cálculo } \\
& \text { podríamos despreciar } d y^{\prime} \Rightarrow F_{p}=\iint_{A} P(x, z) d x^{\prime} \text { " }
\end{aligned}
$$

Despreciar una de las variables que participan del elemento diferencial de superficie, anula la fuerza actuando sobre la placa, pero no simplifica la variable de integración por sí sola, lo cual es un error conceptual grave no atribuible a los contenidos del curso. Todo lo contrario. Este tipo de errores se arrastran claramente a problemas de operatoria y conceptos provenientes de los cursos del tronco de matemáticas.

\section{Extracto 5}

En el escenario uniformemente acelerado, un estudiante propone un esquema de cálculo de la fuerza hidrostática en el escenario uniformemente acelerado. El estudiante, sin embargo, adopta la ecuación (5) que corresponde a la situación estática lo que es otro error serio. Aquí se evidencia un problema más severo, donde este alumno y otros que respondieron usando la misma expresión, no lograron conceptualizar adecuadamente el enunciado del ejercicio, revelando entonces problemas cognitivos asociados a la percepción y organización de la información. Si bien, el alumno reconoce la meta (calcular la fuerza hidrostática y con ello la fuerza sobre el sello), pretende llegar a ella saltándose etapas del razonamiento lógico y violando así, los principios físicos que gobiernan el problema. Esta mezcla errónea de conceptos, sumado a esquemas de resolución escuetos confluyen a una solución inexacta y poco comprensible para el evaluador, desembocando en una mala evaluación y calificación.

\section{DISCUSION}

Todos los extractos anteriores ilustran que el proceso de corrección de evaluaciones es un sujeto difícil y subjetivo, pues quien evalúa debe interpretar lo que tratan de decir los alumnos, en lugar de limitarse a corregir lo estrictamente escrito en la respectiva hoja de respuesta. Siguiendo los planteamientos de Zuñiga-Silva (2007, 2018), Duval (2006) y Radford y André (2009), se infiere de los resultados que los alumnos tienden a presentar de forma sistemática sesgos de pensamiento y lagunas cognitivas cuya raíz parece provenir de falencias en el razonamiento matemático y algorítmico que deberían ser superados en los primeros dos años de la carrera de ingeniería. Siguiendo la teoría de Feuerstein (citada por Zuñiga-Silva, 2007 y 2018), al parecer los mayores problemas cognitivos ocurren en la fase de entrada y de elaboración, donde la capacidad de 
pensamiento hipotético y la conducta comparativa parecen verse severamente mermados y en consecuencia, limitando la posibilidad de dar una salida al desafío. En un intento por fortalecer el desarrollo de habilidades orientada a la resolución de problemas conceptuales de Mecánica de Fluidos, Ghosh (2011) también ha dado cuenta de tales lagunas lo que reafirma los resultados del presente estudio.

Varias estrategias se han planteado para reducir tales debilidades. Aznar y Leyton (2017) sugieren que esto podría lograrse desarrollando estrategias pedagógicas orientadas al desarrollo de habilidades de pensamiento crítico en etapas iniciales de la formación universitaria. En este punto es conveniente precisar que, luego de consultar evaluaciones (guías de ejercicios, controles y pruebas) de cursos de ciencias básicas de la carrera (esencialmente cursos de física y matemáticas), se evidencia que el aprendizaje de los alumnos se ha conducido en la modalidad orientada a la resolución de problemas. Sin embargo, dicha modalidad parece haberse focalizado en la aplicación y repetición de fórmulas y no en el desarrollo de habilidades para lograr desestructurar un problema, un elemento clave para encontrar una salida al ejercicio aquí propuesto. Ghosh (2011) plantea además la idea de diseñar cursos electivos como formación complementaria a los cursos del eje de formación disciplinar, de forma de ayudar a retener y profundizar los contenidos en el área de la mecánica de fluidos. Por otro lado, Rahman (2017) y Webster et al. (2016) reportaron buenos resultados en la mejora de habilidades analíticas orientadas al desarrollo de problemas en esta área, pero en un esquema de aula invertida aportando material audiovisual a los alumnos antes del desarrollo de la clase presencial.

En definitiva, todo lo anterior parece confluir hacia la necesidad de fortalecer las habilidades asociadas al razonamiento de cursos de ciencias naturales y exactas en etapas iniciales de la formación ingenieril, quizás pasando de un esquema de repetición de procedimientos y aplicación directa de fórmulas a otro donde los alumnos se vean conminados a usar estos conocimientos como una herramienta para desestructurar problemas de mayor complejidad, construyendo estrategias de solución que tiendan a favorecer el razonamiento crítico. En este sentido, de acuerdo a Kassymova et al (2020) el desarrollo de metodologías de aprendizaje orientado a la resolución de problemas sigue siendo una herramienta útil de preparación aun cuando los alumnos se encuentren ahora en un ambiente de carácter virtual, ofreciendo un camino válido para que los alumnos exploten dicho potencial de pensamiento, pero más importante aún para que los estudiantes más desfavorecidos en su funcionamiento cognitivo en estas áreas logren transitar con éxito hacia cursos superiores y mantenerse en estas carreras (García et al. 2018; Armstrong y Croft, 1999).

Las variables motivacionales también podrían cobrar fuerza en este contexto dentro de un esquema virtual. Esto ocurre pues hoy en día se percibe una desmotivación de los alumnos por empaparse de su profesión, de los procesos educativos y de los conocimientos que se le imparten (Anaya-Durand y Anaya-Huertas, 2010), sugiriendo la necesidad de replantear el triángulo pedagógico en el contexto virtual aplicado a la enseñanza en el campo de la ingeniería y ciencias.

Trasladar estos desafíos al aula virtual es una tarea de gran envergadura, respecto de la cual Junus et al (2019) sugiere que el uso intensivo de los foros de discusión tiene el potencial de facilitar y estimular el aprendizaje colaborativo de los alumnos, junto con su pensamiento crítico. El uso de estos foros, junto con la necesidad de mantener permanentemente disponibles apuntes y recursos de estudio online, es una opinión también compartida por Kassymova et al. (2020) para el éxito de este tipo de expediciones pedagógicas. En este sentido, el presente artículo ha desnudado un pobre uso de esta herramienta por parte de los alumnos sugiriendo que muchos de ellos prefieren retener sus dudas y arriesgarse a ser mal evaluados, antes que compartir y discutir vías alternativas de solución con el resto del grupo y el propio equipo docente. Este elemento también pudo haber contribuido significativamente al pobre desempeño de los estudiantes en la segunda parte de la tarea.

Finalmente, el presente estudio también ha tenido como espíritu entregar luces de alerta a la comunidad universitaria y al mismo tiempo una oportunidad de mejora que los docentes pueden explotar en las plataformas virtuales, en virtud que estos hallazgos ocurren en un ambiente en ausencia de la presencia física del equipo docente (profesor y ayudantes) y donde, además, se propende el trabajo colaborativo y la discusión entre pares. La ausencia de supervisión presencial directa en este tipo de sesiones son elementos que pueden generar impactos no deseados en el ambiente de aprendizaje.

\section{CONCLUSIONES}

De acuerdo al trabajo presentado y a los resultados obtenidos, se pueden plantear las siguientes conclusiones principales:

1. De los resultados del presente estudio se desprende que los alumnos responden las dificultades de la tarea con notable destreza cuando las demandas cognitivas del ejercicio son de baja intensidad. En cambio, cuando dicha demanda aumenta solo un porcentaje marginal del grupo logra obtener resultados aceptables y destacados. 
2.- Casi $2 / 3$ del grupo exhiba un pobre desempeño en la segunda parte de la tarea, revelando que existen problemas cognitivos asociados probablemente a la comprensión, pensamiento hipotético y conducta comparativa que no han sido adecuadamente explotados o potenciados en el tránsito desde cursos de formación inicial a cursos de especialidad.

3.- La presencia física del maestro en el salón de clases juega un rol primordial en el proceso de aprendizaje. En el contexto virtual dicha figura se desvanece y el profesor se transforma en un facilitador de información a los estudiantes. Los alumnos parecen no acomodarse del todo a esta nueva figura, especialmente cuando son conminados a resolver problemas de manera autónoma o solo con material de apoyo.

4.- En este sentido, se hace necesaria realizar un acompañamiento online mucho más intenso con los alumnos, incrementando las horas efectivas de labor docente variable que, al parecer, no ha sido adecuadamente conmensurada en este nuevo escenario de aprendizaje a nivel nacional.

5.- El uso de plataformas virtuales de aprendizaje, esconde varias herramientas que podrían potenciar el aprendizaje de cursos más especializados. Esta situación atañe particularmente al uso de foros de discusión de carácter asincrónico.

6.- El presente estudio desnuda un pobre uso de esta herramienta por parte de los alumnos, aun cuando el docente a cargo creó y facilitó todos los canales para promover dicha comunicación.

7.- Tal vez un uso más intenso de este tipo de herramientas podría ayudar a subsanar dudas conceptuales no solo del ejercicio en particular, sino además lagunas cognitivas que los alumnos arrastran a partir de los contenidos vistos en clases y que tal vez por alguna razón relacionada con variables emocionales, estos tienden a guardarse para sí, dificultando la efectividad de aprendizaje.

\section{REFERENCIAS}

Absi, R., Nalpas C., Dufour F., Huet D., Bennacer R. y Absi, T., Teaching Fluid Mechanics for Undergraduate Students in Applied Industrial Biology: from Theory to Atypical Experiments. arXiv:1106.0656v1, International Journal of Engineering Education, 27(3), 550-558 (2011)

Anaya-Durand, A. y Anaya-Huertas, C., ¿Motivar para aprobar o para aprender? Estrategias de motivación del aprendizaje para los estudiantes. Tecnología, Ciencia, Educación, ISSN: 0186-6036, 25(1), 5-14 (2010)

Armstrong, P. y Croft, A., Identifying the Learning Needs in Mathematics of Entrants to Undergraduate Engineering Programmes in an English University. https://doi.org/10.1080/03043799908923538, European Journal of Engineering Education, 24(1), 59-71 (1999)

Aznar, I. y Laiton, I., Desarrollo de Habilidades Básicas de Pensamiento Crítico en el Contexto de la Enseñanza de la Física Universitaria. DOI: 10.4067/S0718-50062017000100008, Formación Universitaria, 10(1), 71-78 (2017)

Báez, A.M., Martínez-López, Y., Pérez, O.L. y Pérez, R. Propuesta de Tareas para el Desarrollo del Pensamiento Variacional en Estudiantes de Ingeniería. DOI: 10.4067/S0718-50062017000300010, Formación Universitaria, 10(3), 93106 (2017)

Chandler, T., Park, Y.S., Levin K.L. y Morse, S., The incorporation of hands-on tasks in an online course: an analysis of a blended learning environment. https://doi.org/10.1080/10494820.2011.593524, Interactive Learning Environments, 21(5), 456-468 (2013)

Duval, R., A Cognitive Analysis of Problems of Comprehension in a Learning of Mathematics. https://doi.org/10.1007/s10649-006-0400-z, Educational Studies in Mathematics, 61, 103-131 (2006)

Fernández, B., Introducción a la Mecánica de Fluidos. 4ª Edición, Ediciones U. Católica de Chile, 432 p. (2013)

Fox, R., McDonald, A. y Pritchard, P., Introduction to Fluid Mechanics. 6th Edition, Wiley \& Sons, 800 p. (2003)

García, M.L. y Benítez, A., Diseño e Implementación de Tareas para Apoyar el Aprendizaje de las Matemáticas. DOI: 10.4067/S0718-50062013000100003, Formación Universitaria, 6(1), 13-20 (2013)

García, M.L., López, A. y Díaz, A., Análisis del Desempeño de Estudiantes en Tareas Matemáticas. Estudio Exploratorio en el Instituto Politécnico Nacional de México. http://dx.doi.org/10.4067/S0718-50062018000500041, Formación Universitaria, 11(5), 41-54 (2018)

Ghosh, A., Teaching Formulation Skills in an Upper Level Fluid Mechanics Course. Proceedings of IMECE2011, ASME International Mechanical Engineering Congress and Exposition, USA (2011)

Huilier, D.G.F., Forty Years' Experience in Teaching Fluid Mechanics at Strasbourg University. https://doi.org/10.3390/fluids4040199, Fluids, 4, 199 (2019) 
Junus, K., Suhartanto, H., y otros 3 autores, The Community of Inquiry model training using the cognitive apprenticeship approach to improve students' learning strategy in the asynchronous discussion forum. Journal of Educators Online, 16(1) (2019)

Kassymova G., Akhmetova A., y otros cuatro autores, E-Learning Environments and Problem-Based Learning. International Journal of Advanced Science and Technology, 29 (7) (2020)

Pal, R., Teaching Fluid Mechanics and Thermodynamics Simultaneously through Pipeline Flow Experiments. https://doi.org/10.3390/fluids4020103, Fluids, 4, 103 (2019)

Radford, L. y André. M., Cerebro, cognición y matemáticas. Revista Latinoamericana de Investigación en Matemática Educativa, ISSN 2007-6819, 12(2), 215-250 (2009)

Rahman A., A Blended Learning Approach to Teach Fluid Mechanics in Engineering. https://doi.org/10.1080/03043797.2016.1153044, European Journal of Engineering Education, 42(3), 252-259 (2017)

Soares, F., Leão, C. P., y otros cinco autores, Interpreting Students' Perceptions in Fluid Mechanics Learning Outcomes. Education in the Knowledge Society, E-ISSN: 2444-8729 ,16(3), 73-90 (2015)

Webster, D. R., Majerich, D. M. y Madden, A. G., Flippin' Fluid Mechanics-Comparison Using Two Groups. Advances in Engineering Education, EISSN-1941-1766, 5(3) (2016)

White, F.M. Fluid Mechanics. 8th Edition, McGraw-Hill, 755 p. (2016)

Zúñiga-Silva, L., El Cálculo en Carreras de Ingeniería: Un Estudio Cognitivo. Revista Latinoamericana de Investigación en Matemática Educativa, 10(1), 145-175 (2007)

Zúñiga-Silva, L., Matemáticas para Ingeniería: un Análisis del Funcionamiento Cognitivo en la Solución de un Problema de Cálculo en Dos Variables. http://dx.doi.org/10.4067/S0718-50062018000500055 , Formación Universitaria, 11(5), 5564 (2018) 
